\title{
La valoración preanestésica del paciente pediátrico durante la pandemia por COVID-19
}

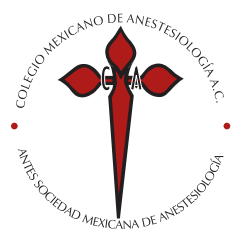

\author{
Preanesthetic assessment of the pediatric patient \\ during the COVID-19 pandemic
}

\author{
Dra. Belém Mejía-Peñaloza,* Dra. Diana Moyao-García ${ }^{\ddagger}$
}

Citar como: Mejía-Peñaloza B, Moyao-García D. La valoración preanestésica del paciente pediátrico durante la pandemia por COVID-19. Rev Mex Anestesiol. 2021; 44 (4): 282-287. https://dx.doi.org/10.35366/100874

RESUMEN. En la pandemia actual por SARS-CoV-2, poca atención se ha puesto a la población pediátrica debido a su baja morbimortalidad. Debido al amplio espectro de presentación de esta enfermedad en los pacientes pediátricos, es necesario reconocer sus diferentes presentaciones clínicas y la importancia de la valoración preanestésica en procedimientos electivos y de urgencia a fin de evitar la exposición del personal de salud a pacientes contagiados y disminuir la morbilidad perioperatoria en el paciente pediátrico con COVID-19.

ABSTRACT. In the current SARS-CoV-2 pandemic, little attention has been paid to the pediatric population due to its low morbidity and mortality. Due to the wide spectrum of presentation of this disease, in pediatric patients, it is necessary to recognize its different clinical presentations and the importance of pre-anesthetic evaluation in elective and emergency procedures in order to avoid the exposure of health personnel with infected patients and to reduce the perioperative morbidity in the pediatric patient with COVID-19.

\section{INTRODUCCIÓN}

E n diciembre de 2019, en Wuhan, China, se informó del descubrimiento del virus SARSCoV-2 y éste rápidamente alcanzó el nivel de pandemia. Sin embargo, la atención hacia el paciente pediátrico quedó en segundo plano, quizá debido a la baja mortalidad de esta población al ser comparada con la adulta.

A causa de que el rango de presentación clínica de la población pediátrica es amplio y el acceso a las pruebas de detección del SARS-CoV-2 permanece limitado, no debería sorprendernos el hecho de que nos encontráramos con más frecuencia a pacientes pediátricos portadores del virus (con o sin síntomas) con el potencial riesgo de infección al personal de salud.

En este contexto, la valoración preanestésica del paciente pediátrico es fundamental y requiere del conocimiento de las formas de presentación de la enfermedad en esta población; sobre todo en los casos en los que se planea una cirugía o procedimiento de urgencia, donde no se puede esperar al resultado de la prueba reacción en cadena de la polimerasa con transcriptasa inversa (RT-PCR por sus siglas en inglés) o en los casos de pacientes sintomáticos con resultado negativo de la prueba.

\section{INFECCIÓN POR COVID-19 EN EL PACIENTE PEDIÁTRICO}

La mediana del período de incubación de la infección por COVID-19 en el paciente pediátrico es de 4-5 días con un rango de hasta 24 días. Con una edad promedio de infección entre los 6-7 años. La mayoría de los niños se recupera en 1-2 semanas después del inicio de la enfermedad ${ }^{(1)}$.

Palabras clave:

Valoración preanestésica, paciente pediátrico, COVID-19.

Keywords:

Pre-anesthetic evaluation, pediatric patient, COVID-19.

* Anestesióloga Pediatra. Médico adscrito al Servicio de Anestesia. Hospital Pediátrico de Coyoacán. México.

‡Anestesióloga Pediatra. Jefa del Servicio de Anestesia y Algología. Hospital Infantil de México Federico Gómez. México.

Correspondencia: Dra. Belém Mejía-Peñaloza Prol. Moctezuma Núm. 18, Col. Del Carmen, 04000, Alcaldía Coyoacán, Ciudad de México, México. E-mail: belemmp24@gmail.com

Recibido: 27-08-2020

Aceptado:18-11-2020

Abreviaturas:

DHL $=$ Deshidrogenasa láctica. ECA-2 = Enzima convertidora de angiotensina 2.

MIS-C = Multi-system inflammatory syndrome in children. PAPR = Powered air purifying respirators. RT-PCR = Reverse transcription polymerase chain reaction (reacción en cadena de la polimerasa con transcriptasa inversa). 
En el estudio de Dong y colaboradores, $21 \%$ de los pacientes pediátricos eran asintomáticos, $58 \%$ tenían enfermedad leve, $19 \%$ enfermedad moderada, $1 \%$ enfermedad grave y $1 \%$ estaban en estado crítico $^{(2)}$. Sin embargo, dependiendo del estudio, existen informes donde la población pediátrica asintomática puede llegar hasta el $90 \%(3)$.

En general, los niños parecen ser menos propensos a desarrollar una forma grave de infección por COVID-19, y comúnmente tienen un curso clínico leve con un buen pronóstico ${ }^{(4)}$. Las razones probables incluyen tener un sistema inmune aún inmaduro, un tracto respiratorio más saludable y menos afecciones subyacentes que los adultos ${ }^{(1)}$. Otras hipótesis incluyen una posible protección cruzada parcial de los anticuerpos producidos debido a frecuentes infecciones por otros coronavirus durante la primera infancia, diferencias en la respuesta inmunológica que resultan en una menor propensión a progresar a un estado hiperinflamatorio o tormenta de citocinas ${ }^{(5)}$.

La expresión diferencial del receptor de enzima convertidora de angiotensina 2 (ECA-2) en niños también se ha hipotetizado como un contribuyente a sus tasas más bajas de infección y padecimiento de la enfermedad más leve. Se descubrió que la expresión del gen ECA-2 es más baja en el epitelio nasal de niños pequeños y la expresión aumenta con la edad, lo que también proporciona un efecto protector contra la adquisición de infección en niños pequeños ${ }^{(6)}$.

\section{PRESENTACIÓN CLÍNICA DEL COVID-19 EN EL PACIENTE PEDIÁTRICO}

En casos sintomáticos, la presentación es típica de las infecciones respiratorias agudas y con frecuencia incluyen fiebre (59\%) y tos (46\%), que pueden ir acompañadas de congestión nasal, secreción nasal, conjuntivitis, faringitis, sibilancias, mialgias y expectoración. La baja saturación de oxígeno de menos del 92\%, disnea, cianosis y mala alimentación son menos frecuentes que en los adultos ${ }^{(1)}$.

Pocos casos tienen una presentación atípica con manifestaciones gastrointestinales, como náuseas, vómitos y diarrea. Se ha demostrado que, cuando están presentes, los síntomas gastrointestinales generalmente anticipan al patrón respiratorio típico. Estudios previos en casos de SARS-CoV-2 demostraron la detección viral en muestras de biopsia intestinal y heces de pacientes recuperados, lo que indica un posible tropismo del tracto gastrointestinal que puede explicar los síntomas extrarrespiratorios y el desprendimiento viral persistente a través de la ruta fecal-oral ${ }^{(7)}$.

Entre los lactantes, los síntomas como irritabilidad, respuesta reducida y mala alimentación podrían ser los principales signos de infección ${ }^{(1)}$.

La prevalencia de enfermedad grave y crítica parece mayor en los lactantes, en especial en niños menores de un año y en niños con enfermedades subyacentes ${ }^{(1)}$.
Otros síntomas sistémicos parecen estar relacionados con la infección, pero su vínculo aún no se ha demostrado. Desde el brote de la pandemia, se han reportado una gran cantidad de erupciones cutáneas, urticaria y vasculitis que afectan las manos y los pies de niños y adolescentes sanos; así como picazón, ardor, dificultad en los movimientos articulares y dolor ${ }^{(6)}$.

Se han informado manifestaciones neurológicas en pacientes con COVID-19 que incluyen enfermedades cerebrovasculares agudas, alteración de la conciencia, disfunción de nervios craneales, encefalopatía necrosante hemorrágica aguda y síndrome de Guillain-Barré. Sin embargo, aún no se conoce la prevalencia de trastornos neurológicos en niños con COVID-19(6).

Se ha hipotetizado la relación entre la infección por COVID-19 y el desarrollo de enfermedades cardíacas en niños. Belhadjer y colaboradores han informado que un gran número de niños con fiebre resultaron positivos para COVID-19 y fueron ingresados en unidades de cuidados intensivos por insuficiencia cardíaca aguda asociada con un estado inflamatorio multisistémico. En la mayoría de los niños, las características clínicas parecían similares a las del síndrome de Kawasaki: fiebre duradera, erupción cutánea, linfadenopatía, activación persistente de la inflamación sistémica y respuesta positiva a la inmunoglobulina intravenosa ${ }^{(8)}$. Al igual que en la infección por COVID-19, el síndrome de Kawasaki se desencadena por la cascada proinflamatoria activada por la respuesta de inmunidad innata. Sin embargo, se necesitan más estudios para establecer la relación patogénica real entre el COVID-19 y síndromes similares al de Kawasaki ${ }^{(1)}$.

También existen reportes de pacientes pediátricos previamente sanos que desarrollaron síndrome de choque tóxico, cuyo único antecedente de relevancia fue una infección previa por COVID-19 ${ }^{(9)}$.

En algunos casos, la infección por SARS-CoV-2 puede resultar en el síndrome inflamatorio multisistémico en niños (MIS-C por sus siglas en inglés). La presentación clínica de MIS-C incluye fiebre, enfermedad grave y la participación de dos o más órganos o sistemas, en combinación con evidencia de inflamación y de infección por SARS-CoV-2 ${ }^{(10)}$.

La infección por COVID-19 también puede desencadenar la aparición de otras enfermedades inmunomediadas, como la trombocitopenia inmune, el síndrome de Evans, y la anemia hemolítica autoinmune ${ }^{(1)}$.

En cuanto a los resultados bioquímicos, los hallazgos de laboratorio de los niños son similares a las infecciones causadas por diferentes coronavirus. El recuento de glóbulos blancos suele ser normal o reducido con disminución de neutrófilos y linfocitos. Puede ocurrir trombocitopenia. Los niveles de procalcitonina y proteína $C$ reactiva (PCR) suelen ser normales. En casos severos, hay niveles elevados de enzimas hepáticas y deshidrogenasa láctica (DHL), así como anormalidades en la coagulación y niveles elevados de dímero $\mathrm{D}^{(5)}$. 
1. ¿El paciente ha sido diagnosticado con COVID-19?

2. ¿Ha estado el paciente en contacto con una persona diagnosticada con COVID-19 en los últimos 14 días?

3. ¿Ha viajado el paciente a zonas con alta incidencia de la enfermedad?

4. ¿Ha viajado el paciente en avión o barco durante los últimos 14 días?

5. ¿Ha tenido el paciente fiebre de $37.3^{\circ} \mathrm{C}$ o más en los últimos 14 días?

6. ¿Alguien en la casa del paciente ha tenido fiebre de $37.3^{\circ} \mathrm{C}$ o más en los últimos 14 días?

7. ¿Ha tenido el paciente algún problema respiratorio reciente, como tos o dificultad para respirar en los últimos 14 días?

8. ¿Ha tenido el paciente tos reciente en los últimos 14 días?

9. ¿Ha tenido el paciente diarrea reciente en los últimos 14 días?

10. ¿Ha tenido el paciente mialgias, rinorrea, malestar, dolor de cabeza, náuseas o vómitos en los últimos 14 días?

11. ¿Ha estado el paciente en contacto con personas con fiebre o problemas respiratorios en los últimos 14 días?

12. ¿Ha participado el paciente recientemente en reuniones o ha tenido contacto cercano con muchas personas que no conoce?

13. ¿Ha perdido el paciente, algún familiar o contactos cercanos la capacidad de oler?

Modificado de: Soneru CN, et al. ${ }^{18}$

En el estudio de De Souza y su equipo se observó una disminución en el recuento de linfocitos en sólo $12.9 \%$ de los niños infectados, en contraste con los adultos, en los cuales $80 \%$ de los casos no graves tienen linfopenia. Por lo tanto, la linfopenia puede no ser un indicador confiable de COVID-19 en niños ${ }^{(11)}$.

Entre los hallazgos radiológicos, con frecuencia se detectan opacidades en vidrio esmerilado, infiltrados mono o bilaterales y pequeños nódulos. En casos severos, las alteraciones radiológicas se difunden, presentándose como un «pulmón blanco». Sin embargo, la evidencia radiológica de neumonía podría estar ausente en el $15-20 \%$ de los niños ${ }^{(1)}$.

Gotzinger y su grupo realizaron un estudio de cohorte multicéntrico, donde incluyeron 582 niños con infección por SARS-CoV-2 confirmada por RT-PCR. Los resultados fueron: mediana de edad de cinco años, proporción de sexos de 1.15 hombres por mujer, 25\% tenían condiciones médicas preexistentes, $62 \%$ fueron ingresados en el hospital, $8 \%$ requirieron ingreso en la UCI, 4\% requirieron ventilación mecánica (mediana de duración siete días), 3\% soporte inotrópico y menos del 1\% oxigenación con membrana extracorpórea. Los factores de riesgo significativos para requerir la admisión en la UCI fueron: ser menores de un mes, sexo masculino, afecciones médicas preexistentes y presencia de signos o síntomas de infección del tracto respiratorio inferior. En este estudio se informó una tasa de letalidad de $0.69 \%{ }^{(12)}$.

\section{COVID-19 EN EL RECIÉN NACIDO}

Las infecciones neonatales por SARS-CoV-2 también son extremadamente raras y, hasta la fecha, no hay evidencia de infección intrauterina causada por transmisión vertical. Como se describe en un informe de caso y una serie de casos, el líquido amniótico, la sangre del cordón umbilical, el hisopo neonatal de la faringe y las muestras de calostro recolectadas de madres infectadas fueron negativas para COVID-19. Sin embargo, la pregunta sigue siendo controvertida, ya que se han detectado anticuerpos IgM en recién nacidos de madres con COVID-19(7).

El diagnóstico de infección neonatal por SARS-CoV-2 debe cumplir con todos los criterios siguientes: 1) al menos un síntoma clínico, que incluye temperatura corporal inestable, baja actividad o mala alimentación, o decaimiento; 2) radiografías de tórax que muestren anormalidades, incluidas opacidades en vidrio esmerilado unilaterales o bilaterales; 3 ) un diagnóstico de infección por SARS CoV-2 en la familia o cuidadores del paciente; y 4) contacto cercano con personas que pueden tener o han confirmado una infección por SARSCoV-2 o pacientes con neumonía inexplicable ${ }^{(13)}$.

Todas las infecciones de SARS-CoV-2 neonatales posibles o confirmadas por laboratorio deben ingresarse en la Unidad de Cuidados Intensivos Neonatales ${ }^{(13)}$.

Si los recién nacidos también presentan síndrome de dificultad respiratoria, se debe considerar la administración de surfactante pulmonar en dosis altas, inhalación de óxido nítrico y ventilación oscilatoria de alta frecuencia ${ }^{(1,5,13)}$.

En los recién nacidos en estado crítico, también se puede considerar la administración intravenosa de glucocorticoides o inmunoglobulinas, reemplazo renal y oxigenación con membrana extracorpórea ${ }^{(1,13)}$.

\section{COVID-19 EN NIÑOS INMUNOSUPRIMIDOS}

Hasta la fecha, hay pocas publicaciones sobre COVID-19 en niños con neoplasias malignas o postoperados de trasplante de células madre hematopoyéticas; los informes y las series de casos de niños con neoplasias malignas y COVID-19 han descrito de manera predominante una enfermedad de leve a moderada. También se han informado hallazgos similares en pacientes con trasplantes de órganos sólidos ${ }^{(6)}$. 
A pesar de estos informes tempranos que resultan tranquilizadores, también han surgido informes poco frecuentes de deterioro clínico rápido en niños inmunocomprometidos con COVID-19, destacando la necesidad de un seguimiento estrecho en estos pacientes debido a un curso clínico impredecible $^{(6)}$.

\section{¿EL PACIENTE PEDIÁTRICO COMO FUENTE DE DISEMINACIÓN DEL COVID-19?}

Aunque no se cuenta con evidencia sólida, los niños han sido identificados como una fuente potencial de diseminación del virus de niños a adultos ${ }^{(14)}$. A pesar de ser casos leves o asintomáticos, la eliminación viral prolongada en las heces y las secreciones nasales hace posible que los niños sean facilitadores de la transmisión del virus ${ }^{(1)}$.

Tabla 2: Signos, síntomas y hallazgos de laboratorio del paciente pediátrico con COVID-19.

Síntomas inespecificos

Respiratorio

Gastrointestinal

Piel

Cardiovascular

$\begin{array}{ll} & \text { y de manos y pies, exantema polimorfo, } \\ & \text { adenopatías cervicales, pancarditis) } \\ & \text { Síndrome de shock séptico: hipotensión, } \\ & \text { erupción eritrodérmica difusa, afectación de } \\ & \text { mucosas y disfunción orgánica multisistémica } \\ & \text { (renal, hepática, hematológica, respiratoria, } \\ & \text { muscular y neurológica) } \\ \text { Musculoesquelético } & \text { Artralgias, mialgias, dificultad en la movilización } \\ \text { Neurológico } & \text { Enfermedad cerebrovascular aguda, } \\ & \text { alteración de la conciencia, disfunción de } \\ & \text { nervios craneales, encefalopatía necrosante } \\ & \text { hemorrágica aguda, síndrome de Guillain-Barré } \\ \text { Inmunológico } & \text { Trombocitopenia inmune, síndrome de Evans, } \\ & \text { anemia hemolítica autoinmune } \\ \text { Síndrome inflamatorio } & \text { Fiebre, enfermedad grave y disfunción de dos } \\ \text { multisistémico en niños } & \text { o más órganos o sistemas, más evidencia de } \\ & \text { inflamación y de infección por SARS-CoV-2 } \\ \text { Radiografía } & \text { Lesiones en parche, consolidaciones, } \\ & \text { opacidades en vidrio esmerilado } \\ \text { Laboratorios } & \text { Cuenta leucocitaria normal (83\%) } \\ & \text { Linfopenia, neutropenia, trombocitopenia } \\ & \text { (raras; } 3 \%) \\ \text { Niveles elevados de enzimas hepáticas y DHL } \\ \text { (raro; } 3 \%) \\ \text { Elevación de PCR, procalcitonina y dímero D } \\ \text { (raro; } 3 \% \text { ) }\end{array}$

$\mathrm{DHL}$ = deshidrogenasa láctica; $\mathrm{PCR}=$ proteína $\mathrm{C}$ reactiva.
En la investigación de Xu y colaboradores ocho de cada 10 niños con SARS-CoV-2 tuvieron hisopos rectales persistentemente positivos, incluso después de que sus pruebas nasofaríngeas fueran negativas ${ }^{(15)}$.

\section{PLANIFICACIÓN PREOPERATORIA}

Dada la amplia variedad de presentaciones clínicas en el paciente pediátrico, la planificación preoperatoria es imprescindible para evitar procedimientos innecesarios. Las cirugías deben evaluarse y se debe garantizar que cumplan con los siguientes criterios: 1 ) potencialmente salvadora de vidas; 2) puede prevenir el deterioro rápido del paciente; 3 ) puede prevenir la discapacidad o disfunción permanente; o 4) la imposibilidad de realizar la cirugía puede conducir a una progresión metastásica o infecciosa ${ }^{(16)}$.

Una vez que la cirugía se considere apropiada, las pruebas preoperatorias para COVID-19 deben realizarse lo antes posible y los resultados deben obtenerse antes de proceder, siempre que el paciente esté clínicamente estable ${ }^{(16)}$.

Está indicado el hisopado nasofaríngeo con RT-PCR realizada dentro de las 48 horas previas a la operación, desafortunadamente la prueba presenta del 30 al $40 \%$ de falsos negativos; sin embargo, es necesaria sobre todo si hay signos sugestivos de infección por COVID-19(17).

«Una prueba negativa no garantiza la ausencia de enfermedad dado el riesgo de falsos negativos y es insuficiente para negar la necesidad de precauciones intraoperatorias sobre todo cuando el procedimiento tiene alto riesgo de aerosolización» ${ }^{(6)}$.

\section{VALORACIÓN PREANESTÉSICA}

En caso de cirugías electivas, se dispone de mayor tiempo para poder definir si la condición del paciente es segura para el personal de salud.

Soneru y colaboradores recomiendan la realización de un cuestionario por teléfono previo al ingreso hospitalario del paciente (Tabla 1). En el caso de que un padre responda Sí a cualquier pregunta, se debe considerar posponer el procedimiento y repetir la evaluación en 14 días, además de indicar al paciente y familiares cercanos que se pongan en cuarentena en casa y notifiquen los síntomas al departamento de salud local. Si el padre de un paciente responde NO a todas las preguntas de detección, la programación de la cirugía puede continuar $^{(18)}$. Sin embargo, hay que recordar que los pacientes que no presentan ningún síntoma pueden tener el virus y ser contagiosos.

En la valoración preanestésica durante el interrogatorio por aparatos y sistemas y la exploración física, se deben buscar signos y síntomas sugestivos de la enfermedad, y complementarlos con la información que nos proporcionen 
Tabla 3: Recomendaciones de la ASA para los profesionales sanitarios que atienden a pacientes con COVID-19 (sospechosos o conocidos).

1. Colocar a los pacientes en una sala de aislamiento de infecciones transmitidas por aire

2. Los profesionales de la salud que ingresen a la habitación deben tomar precauciones de contacto y de transmisión aérea, incluida la protección ocular

3. El equipo de protección personal (EPP) que se debe usar incluye:

a. Mascarilla N95, para la cual se ha realizado una prueba de ajuste, o un respirador con purificador de aire motorizado (PAPR)

b. Un protector facial o gafas

c. Bata

d. Guantes

4. La higiene de manos es fundamental antes de ponerse y después de quitarse el EPP; se puede realizar usando desinfectantes para manos a base de alcohol o lavándose las manos con agua y jabón (al menos 20 segundos)

5. Tener mucho cuidado al quitarse y desecharse el EPP para minimizar el riesgo de autocontaminación. Considere seriamente observar los procedimientos correctos para ponerse y quitarse el EPP y luego ensayar estos procedimientos antes de la atención directa al paciente

ASA = American Society of Anesthesiologists (Sociedad Americana de Anestesiologia).

Modificado de: Soneru $\mathrm{CN}$, et al.18

los estudios de laboratorio y gabinete solicitados (Tabla 2). Todo esto con el objetivo de que nos alerten de un posible contagio por parte del paciente a pesar de haber pasado la prueba del cuestionario.

Durante la anestesia, los procedimientos que se recomienda realizar deberían ser aquéllos que minimicen la tos y la presión alta en las vías respiratorias ${ }^{(19)}$.

Se deben seguir las recomendaciones de la Sociedad Americana de Anestesiología (ASA) (Tabla 3) y reservar ciertos quirófanos y máquinas de anestesia para su uso sólo en casos COVID-19; además las trampas de agua, el circuito de anestesia, los filtros de intercambio de calor y humedad y los filtros de dióxido de carbono deben cambiarse después de cada paciente. En la medida de lo posible, durante la pandemia, las cirugías deben realizarse bajo anestesia regional, ya que tiene los beneficios de preservar la función respiratoria y evitar el uso de aerosoles y, por lo tanto, la transmisión viral ${ }^{(19,20)}$.

\section{MORBIMORTALIDAD POSTOPERATORIA EN EL PACIENTE CON COVID-19}

A pesar de que no se dispone de información sobre el impacto de la morbimortalidad perioperatoria en el paciente pediátrico con COVID-19, la información que tenemos de la población adulta nos alienta a considerar posponer los procedimientos que no sean urgentes y promover el tratamiento no quirúrgico para retrasar o evitar la necesidad de cirugía en el momento de la infección ${ }^{(21)}$. Esto debido a que se observa un aumento de las complicaciones postoperatorias, sobre todo las respiratorias y trombóticas, así como un aumento de la mortalidad ${ }^{(22)}$.

El COVID-19 grave se asocia con un marcado estado inflamatorio y protrombótico. Estos procesos patológicos se agravan con la cirugía y la inmovilización, lo que conduce a una tormenta perfecta que perjudica los buenos resultados postoperatorios ${ }^{(23)}$.

Las complicaciones pulmonares postoperatorias, definidas como neumonía, síndrome de dificultad respiratoria aguda o ventilación mecánica postoperatoria inesperada, ocurren en la mitad de los pacientes con infección perioperatoria por SARS-CoV-2 y se asocian con una alta mortalidad (aproximadamente el $50 \%$ de casos) ${ }^{(21)}$.

La tasa de mortalidad global descrita en adultos con SARS-CoV-2 son de $18.9 \%$ en casos electivos, $25.6 \%$ de emergencia, $16.3 \%$ sometidos a cirugía menor y $26.9 \%$ en pacientes sometidos a cirugía mayor ${ }^{(21)}$.

\section{REFERENCIAS}

1. Lassandro G, Palladino V, Amoruso A, Palmieri VV, Russo G, Giordano P. Children in coronaviruses' wonderland: what clinicians need to know. Mediterr J Hematol Infect Dis. 2020;12:e2020042.

2. Dong Y, Mo X, Hu Y, Qi X, Jiang F, Jiang Z, et al. Epidemiology of COVID-19 among Children in China. Pediatrics. 2020;145:e20200702.

3. Parikh M, Fiadjoe J, Black S. Emergency surgery during the COVID-19 pandemic. Paediatr Anaesth. 2020;30:728-729.

4. Parri N, Lenge M, Buonsenso D; Coronavirus Infection in Pediatric Emergency Departments (CONFIDENCE) Research Group. Children with Covid-19 in pediatric emergency departments in Italy. N Engl J Med. 2020;383:187-190.
5. Zimmermann P, Curtis N. Coronavirus infections in children including COVID-19: an overview of the epidemiology, clinical features, diagnosis, treatment and prevention options in children. Pediatr Infect Dis J. 2020;39:355-368.

6. Rajapakse N, Dixit D. Human and novel coronavirus infections in children: a review. Paediatr Int Child Health. 2021;41:36-55.

7. Liguoro I, Pilotto C, Bonanni M, Ferrari ME, Pusiol A, Nocerino A, et al. SARS-COV-2 infection in children and newborns: a systematic review. Eur J Pediatr. 2020;179:1029-1046.

8. Belhadjer Z, Méot M, Bajolle F, Khraiche D, Legendre A, Abakka S, et al. Acute heart failure in multisystem inflammatory syndrome in children in the context of global SARS-CoV-2 pandemic. Circulation. 2020;142:429-436. 
9. Greene AG, Saleh M, Roseman E, Sinert R. Toxic shock-like syndrome and COVID-19: a case report of multisystem inflammatory syndrome in children (MIS-C) [published online ahead of print, 2020 Jun 6]. Am J Emerg Med. 2020;S0735-6757(20)30492-7.

10. Nakra NA, Blumberg DA, Herrera-Guerra A, Lakshminrusimha S. Multi-system inflammatory syndrome in children (MIS-C) following SARS-CoV-2 infection: review of clinical presentation, hypothetical pathogenesis, and proposed management. Children (Basel). 2020;7:69.

11. de Souza TH, Nadal JA, Nogueira RJN, Pereira RM, Brandao MB. Clinical manifestations of children with COVID-19: A systematic review. Pediatr Pulmonol. 2020;55:1892-1899.

12. Gotzinger F, Santiago-García B, Noguera-Julián A, Lanaspa M, Lancella L, Calo Carducci FI, et al. COVID-19 in children and adolescents in Europe: a multinational, multicentre cohort study. Lancet Child Adolesc Health. 2020;4:653-661.

13. Hong H, Wang Y, Chung HT, Chen CJ. Clinical characteristics of novel coronavirus disease 2019 (COVID-19) in newborns, infants and children. Pediatr Neonatol. 2020;61:131-132. doi: 10.1016/j.pedneo.2020.03.001.

14. Nathan N, Prevost B, Corvol H. Atypical presentation of COVID-19 in young infants. Lancet. 2020;395:1481.

15. Xu Y, Li X, Zhu B, et al. Characteristics of pediatric SARS $\square \mathrm{CoV} \square 2$ infection and potential evidence for persistent fecal viral shedding. Nat Med. 2020;26:502 $\square 505$.

16. Francom CR, Javia LR, Wolter NE, Lee GS, Wine T, Morrissey T, et al. Pediatric laryngoscopy and bronchoscopy during the COVID-19 pandemic: A four-center collaborative protocol to improve safety with perioperative management strategies and creation of a surgical tent with disposable drapes. Int J Pediatr Otorhinolaryngol. 2020;134:110059.

17. Leboulanger N, Sagardoy T, Akkari M, Ayari-Khalfallah S, Celerier C, Fayoux P, et al. Guidelines of the French Association of Pediatric Otorhinolaryngology (AFOP) and French Society of Otorhinolaryngology (SFORL). Eur Ann Otorhinolaryngol Head Neck Dis. 2020;137:177-181. doi: 10.1016/j.anorl.2020.04.010.

18. Soneru CN, Nunez K, Petersen TR, Lock R. Anesthetic concerns for pediatric patients in the era of COVID-19. Paediatr Anaesth. 2020;30:737-742. doi: 10.1111/pan.13924.

19. Yamakage M. Anesthesia in the times of COVID-19. J Anesth. 2021;35:325-327.

20. Thampi S, Yap A, Fan L, Ong J. Special considerations for the management of COVID-19 pediatric patients in the operating room and pediatric intensive care unit in a tertiary hospital in Singapore. Paediatr Anaesth. 2020;30:642-646. doi: 10.1111/pan.13863.

21. COVIDSurg Collaborative. Mortality and pulmonary complications in patients undergoing surgery with perioperative SARS-CoV-2 infection: an international cohort study. Lancet. 2020;396:27-38.

22. Doglietto F, Vezzoli M, Gheza F, Lussardi GL, Domenicucci M, Vecchiarelli L, et al. Factors Associated with surgical mortality and complications among patients with and without coronavirus disease 2019 (COVID-19) in Italy. JAMA Surg. 2020;155:691-702. doi: 10.1001/ jamasurg.2020.2713.

23. Myles PS, Maswime S. Mitigating the risks of surgery during the COVID-19 pandemic. Lancet. 2020;396:2-3. 